\title{
Altered Visual Experience Induces Instructive Changes of Orientation Preference in Mouse Visual Cortex
}

\author{
Anne K. Kreile, Tobias Bonhoeffer, and Mark Hübener \\ Department of Cellular and Systems Neurobiology, Max Planck Institute of Neurobiology, 82152 Martinsried, Germany
}

\begin{abstract}
Stripe rearing, the restriction of visual experience to contours of only one orientation, leads to an overrepresentation of the experienced orientation among neurons in the visual cortex. It is unclear, however, how these changes are brought about. Are they caused by silencing of neurons tuned to non-experienced orientations, or do some neurons change their preferred orientation? To address this question, we stripe-reared juvenile mice using cylinder lens goggles. Following stripe rearing, the orientation preference of cortical neurons was determined with two-photon calcium imaging. This allowed us to sample all neurons in a given field of view, including the non-responsive ones, thus overcoming a fundamental limitation of extracellular electrophysiological recordings. Stripe rearing for 3 weeks resulted in a clear overrepresentation of the experienced orientation in cortical layer $2 / 3$. Closer inspection revealed that the stripe rearing effect changed with depth in cortex: The fraction of responsive neurons decreased in upper layer $2 / 3$, but changed very little deeper in this layer. At the same time, the overrepresentation of the experienced orientation was strongest in lower layer $2 / 3$. Thus, diverse mechanisms contribute to the overall stripe rearing effect, but for neurons in lower layer $2 / 3$ the effect is mediated by an instructive mechanism, which alters the orientation tuning of individual neurons.
\end{abstract}

\section{Introduction}

Neurons in the visual cortex respond selectively to basic features of visual stimuli. During a critical period early in life (Hubel and Wiesel, 1970; Gordon and Stryker, 1996) and, to a lesser degree, in adulthood (Antonini et al., 1999; Sawtell et al., 2003; Tagawa et al., 2005; Hofer et al., 2006), these response properties are plastic and can be shaped by visual input. As a point in case, orientation selectivity, neurons' specific responsiveness to the orientation of a bar in visual space, depends on visual experience to fully mature (Blakemore and Van Sluyters, 1975; Crair et al., 1998; White et al., 2001; Wang et al., 2010) and can be modified by stripe rearing, the procedure of raising animals in a visual environment containing contours of only one orientation (Blakemore and Cooper, 1970; Hirsch and Spinelli, 1970). Stripe rearing has been shown to cause a prominent overrepresentation of the experienced orientation using single-unit recordings (Hirsch and Spinelli, 1970; Blasdel et al., 1977; Sengpiel et al., 1999) and intrinsic imaging (Sengpiel et al., 1999; O’Hashi et al., 2007).

However, it is not well understood which specific aspect of the visual input is instrumental for plasticity of orientation selectivity. In particular, do visual stimuli act in a specific and instructive fashion such that neurons actively change their preferred orientation toward the experienced one (Sengpiel et al., 1999), or does

Received April 29, 2011; revised July 14, 2011; accepted Aug. 2, 2011.

Author contributions: A.K.K., T.B., and M.H. designed research; A.K.K. performed research; A.K.K. analyzed data; A.K.K., T.B., and M.H. wrote the paper.

We thank Boehringer Ingelheim Fonds, the Max Planck Society, and the Max Planck Research School for Molecular and Cellular Life Sciences for supporting this work.

Correspondence should be addressed to Mark Hübener, Department of Cellular and Systems Neurobiology, Max Planck Institute of Neurobiology, Am Klopferspitz 18,82152 Martinsried, Germany. E-mail: mark@neuro.mpg.de. DOI:10.1523/JNEUROSCI.2143-11.2011

Copyright $\odot 2011$ the authors $\quad 0270-6474 / 11 / 3113911-10 \$ 15.00 / 0$ visually evoked activity have a permissive role, simply causing neurons that are not driven by the experienced orientation to lose their responsiveness (Stryker et al., 1978)? In the case of permissive changes, one would thus expect to find a reduced fraction of responsive neurons after stripe rearing. In contrast, no change in the number of responsive cells would strongly point to an instructive mechanism. Determining the fraction of responsive neurons, however, is not trivial as silent neurons are not detected with extracellular single-cell recordings, and intrinsic imaging cannot resolve signals from single cells. Therefore we used in vivo two-photon calcium imaging (Stosiek et al., 2003), a technique which allows morphological detection of non-responsive neurons, and which is well suited to determine the orientation preference of many individual neurons in an unbiased fashion. To alter cortical orientation preference in mouse visual cortex, we adapted a stripe rearing protocol which uses skull-mounted goggles with cylindrical lenses to remove all but one orientation from the visual input (Freeman and Pettigrew, 1973; Sengpiel et al., 1999; O’Hashi et al., 2007; Tanaka et al., 2007).

Using this approach, we observed a clear overrepresentation of the experienced orientation after stripe rearing in layer $2 / 3$ of mouse visual cortex. While the overall fraction of responsive neurons decreased slightly, this effect varied with depth in layer 2/3: Responsiveness dropped among neurons in the uppermost tier of layer $2 / 3$, but remained unchanged in lower layer $2 / 3$. At the same time, neurons in lower layer $2 / 3$ showed a clear shift of orientation preference toward the experienced orientation, indicating that the effect in this neuronal population is largely instructive.

\section{Materials and Methods}

Stripe rearing. All experimental procedures were performed in compliance with institutional guidelines of the Max Planck Society and the local government (Regierung von Oberbayern). Goggles were mounted on the 
skull of male C57BL/6 mice (Fig. $1 A, B$ ) at postnatal day 25 (P25) for 19-23 d. To this end, mice were anesthetized with a mixture of fentanyl $(0.05 \mathrm{mg} / \mathrm{kg})$, midazolam $(5.0 \mathrm{mg} / \mathrm{kg})$, and medetomidine $(0.5 \mathrm{mg} / \mathrm{kg})$. A headbar with two threaded holes was implanted to the skull using dental cement (iCEM Self Adhesive, Heraeus Kulzer) after roughening the bone surface with a gel containing $35 \%$ phosphoric acid (GlumA Etch 35 Gel, Heraeus Kulzer) for $10 \mathrm{~s}$. Goggles were individually adjusted to the mouse's head shape and screwed to the headbar. Blinkers restricted the mouse's field of view to the lenses. The goggles contained cylinder lenses made from acrylic (Findeis $\mathrm{GmbH}$ ) with a refractive power of 167 diopters (dpt) or, for control purposes, acrylic lenses with $0 \mathrm{dpt}$.

Mice wearing goggles were kept in cages with striped walls. The stripe pattern had the same orientation as the cylinder lenses. Cage walls of mice wearing control goggles displayed all orientations. Another control group not wearing goggles was kept under standard housing conditions.

Two-photon calcium imaging. To measure the activity of neurons in primary visual cortex (V1), animals were anesthetized using a mixture of fentanyl $(0.05 \mathrm{mg} / \mathrm{kg})$, midazolam $(5.0 \mathrm{mg} / \mathrm{kg})$, and medetomidine $(0.5 \mathrm{mg} / \mathrm{kg})$. Anesthesia was maintained by reinjecting onefifth of the initial dose after $2 \mathrm{~h}$. After removing the goggles, a thin layer of cream (Isoptomax) was applied to the eyes to prevent dehydration during surgery. After attaching a headplate to the skull, a trepanation was performed to expose the cortical surface, which was kept moist with artificial CSF (ACSF; containing, in mM: $125 \mathrm{NaCl}, 5 \mathrm{KCl}, 10$ glucose, 10 HEPES, $2 \mathrm{MgSO}_{4}$, and $2 \mathrm{CaCl}_{2}, \mathrm{pH}$ 7.4). The calciumsensitive dye Oregon Green 488 BAPTA-1, AM (OGB-1, Invitrogen) was dissolved in $4 \mathrm{ml}$ of DMSO containing 20\% Pluronic, and further diluted (1:11) in dye buffer (containing, in mм: $150 \mathrm{NaCl}, 2.5 \mathrm{KCl}$, and $10 \mathrm{HEPES}, \mathrm{pH} 7.4$ ) to a final concentration of $\sim 0.9 \mathrm{~mm}$. The astrocyte marker sulforhodamine 101 (SR101, Invitrogen; Nimmerjahn et al., 2004) was added, yielding a final concentration of $40 \mu \mathrm{M}$. The dye solution was delivered to the visual cortex by pressure injection at a depth of 200-300 $\mu \mathrm{m}$ using a micropipette (3-5 M $\Omega, 8-12$ psi, 2-3 min). After the dye injection, the cortex was sealed with $2 \%$ agarose in saline and a coverslip.

Cells were imaged at nine depth levels with $20 \mu \mathrm{m}$ intervals in random order, between 180 and $340 \mu \mathrm{m}$ below the pial surface in monocular V1. Using a custom-built two-photon microscope equipped with a SpectraPhysics Mai Tai femtosecond laser and a $40 \times$ water-immersion objective (0.8 NA, Olympus), a $180 \times 180 \mu \mathrm{m}$ field of view was scanned at $2 \mathrm{~Hz}$ and $256 \times 256$ pixel resolution. Both dyes were exited at $830 \mathrm{~nm}$. The emitted light was split by a $585 \mathrm{~nm}$ long-pass filter and detected through a 525/50 nm bandpass filter (OGB-1) and a $680 \mathrm{~nm}$ short-pass filter (SR101, AHF Analysentechnik).

Neurons were stimulated with moving gratings of 0.03 cycles per degree (cpd) spatial frequency and $1.5 \mathrm{~Hz}$ temporal frequency; 16 directions were displayed in a pseudo-randomized manner. In a separate dataset, we used spatial frequencies of $0.03-0.1 \mathrm{cpd}$ and temporal frequencies of $1.5-3 \mathrm{~Hz}$. The complete stimulus sequence was repeated four times. The correct placement of the monitor with respect to the receptive fields of the imaged neurons was confirmed using a flickering retinotopy mapping stimulus. Location of the imaging site in monocular V1 was tested by stimulating the ipsilateral eye.
Data analysis. Data were analyzed using MATLAB (MathWorks) and Image $(\mathrm{NIH})$. Image sequences were corrected for tangential drift and small movements caused by heart beat and breathing. Recordings with significant brain movements or vertical drift were excluded from further analysis. Neuronal cell bodies were detected semiautomatically as regions of interest using a set of morphological filters for cell intensity, size, and shape, and subsequently confirmed by visual inspection. Astrocytes were identified by SR101-staining and removed from the dataset. Baseline fluorescence was computed as the mean $\Delta F / F$ of six $500 \mathrm{~ms}$ frames immediately preceding stimulus onset. Cells were considered responsive if the response to any of the stimuli was significantly different from the baseline (ANOVA at $p<0.001$ ). Cells were considered orientationselective if the response to at least one of the 16 directions was significantly different from the response to all other directions (ANOVA at $p<0.001$ ). To determine orientation tuning, a Gaussian was fitted to the mean amplitudes of fluorescence changes evoked by gratings of eight equally spaced orientations moving in both directions. The preferred orientation was defined as the peak, and tuning width as the full width at half-height (FWHH) of the Gaussian. The median normalized residuals of the Gaussian fit were 0.0030 for control and 0.0023 for stripe-reared mice ( $p<0.001$, Wilcoxon rank sum test). To ensure that the slightly better fits of tuning curves obtained from stripe-reared mice did not influence the data analysis in a systematic fashion, we subsampled the data derived from control mice such that the distribution of normalized residuals was statistically indistinguishable from that in stripe-reared mice. The distribution of preferred orientations in control mice was not 
A

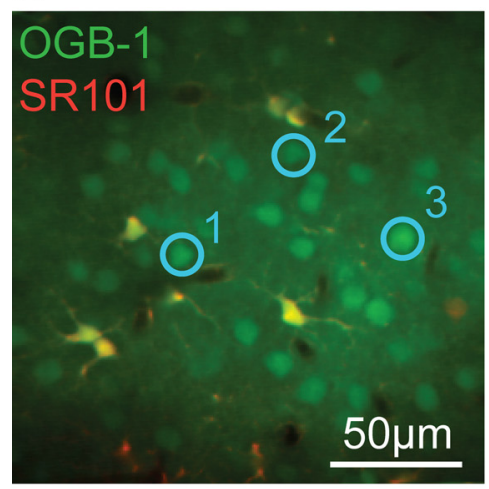

C
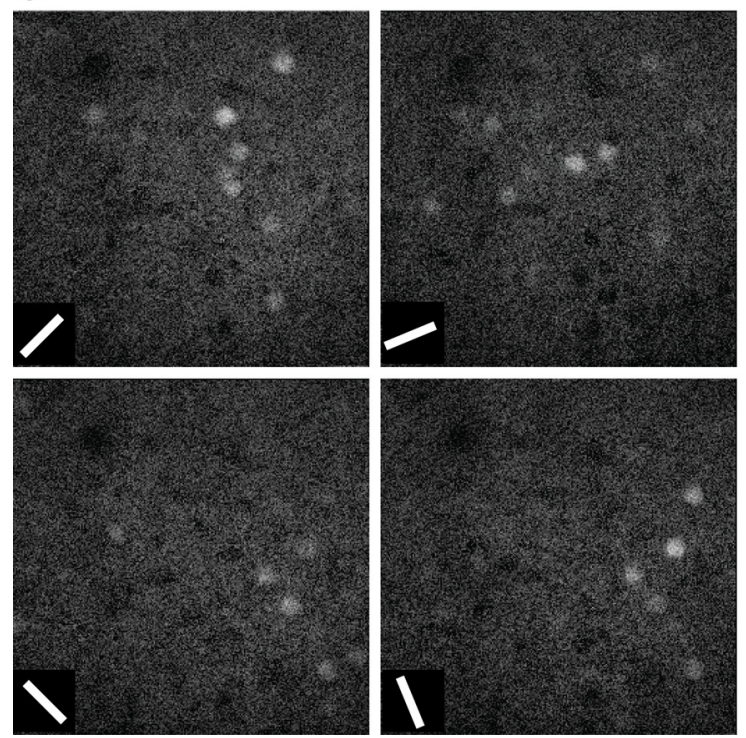

B
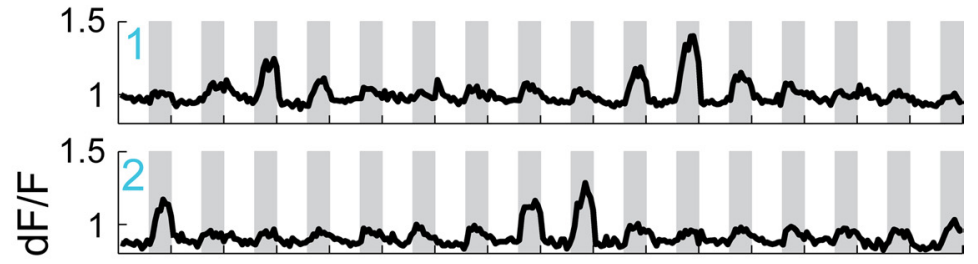

1.5

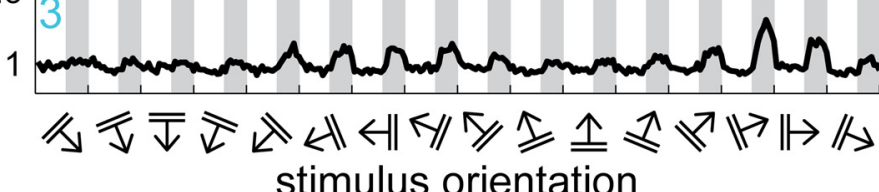

Figure 2. Two-photon calcium imaging of orientation selectivity. A, Optical section at $280 \mu \mathrm{m}$ depth stained with 0GB-1, AM (green) and astrocyte marker sulforhodamine 101 (red); (B) Average $\Delta F / F$ time courses of three representative neurons during stimulus presentation; stimulus orientation and direction are indicated by symbols below; $(C)$ Response maps $(\Delta F / F)$ for eight stimulus orientations, indicated by white bars; all maps are scaled to the same maximum and minimum value.

affected by this procedure, and further data analysis confirmed the presence of a significant stripe rearing effect compared with the subsampled control data.

For display purposes and further statistical analysis, the distribution of orientation preferences was pooled into eight bins, each $22.5^{\circ}$ wide, and centered on the eight orientations used as visual stimuli. The fraction of neurons preferring one of the eight orientations was then normalized either to the total number of orientation-selective neurons or to the total number of detected neurons. If not indicated otherwise, data are shown as mean \pm SEM. For quantification of the stripe rearing effect and analysis of the fraction of neurons tuned to horizontal and vertical orientations, the orientations of interest were pooled together with the two neighboring bins, spanning $67.5^{\circ}$ in total. The stripe rearing effect was quantified as the "specific effect," which is the increase in the experienced orientation plus the decrease in the orthogonal orientation relative to control mice. The "horizontal bias" was calculated as the difference between the fraction of neurons preferring horizontal and the fraction of neurons preferring vertical orientations. For statistical comparisons, a $t$ test or a Wilcoxon rank sum test (for tuning width and amplitude) was performed unless indicated otherwise.

\section{Results}

We induced plasticity of orientation selectivity in mouse visual cortex with a stripe rearing paradigm using the spatial filtering effect of cylindric lenses (Fig. 1C) with a refractive power of
$167 \mathrm{dpt}$. Lenses were mounted on goggles (Fig. 1A) which were fixed to the mice's skulls (Fig. 1B) (O'Hashi et al., 2007). Orientation tuning of individual layer $2 / 3$ neurons in monocular primary visual cortex of stripe-reared and control mice was assessed with two-photon calcium imaging using OGB1-AM bolus loading (Fig. 2A-C) (Stosiek et al., 2003). Astrocytes were identified by Sulforhodamine 101 staining (Fig. 2A) (Nimmerjahn et al., 2004) and excluded from the dataset post hoc.

Stripe rearing shifts the distribution of orientation preference toward the experienced orientation

Mice were stripe-reared using goggles from postnatal day 25 onwards for $\sim 3$ weeks. To provide a high contrast visual input, the walls of the cages were covered with stripes. Control mice kept under standard housing conditions showed a modest overrepresentation of the cardinal orientations $\left(0^{\circ}\right.$ and $\left.90^{\circ}\right)$ with a predominance of horizontally $\left(0^{\circ}\right)$ tuned neurons [Fig. $3 A$; fraction of neurons preferring horizontal and adjacent orientations (see Materials and Methods): $14.6 \pm 0.9 \%$, vertical: $10.7 \pm 1.0 \%, p<0.05$, $t$ test, $n=8$ mice], similar to what has been described in ferrets (Coppola et al., 1998a) and cats (Li et al., 2003). On average, $69 \pm 6 \%$ of the detected neurons were responsive (see Materials and Methods) to orientated gratings, of which $64 \pm 4 \%$ were orientation- 
selective (see Materials and Methods). Tuning width was broadest in vertically and narrowest in horizontally tuned neurons $\left[\right.$ median $\left(0^{\circ}\right)=31^{\circ}$, median $\left(90^{\circ}\right)=47^{\circ}$, $p<5 \times 10^{-19}$, Wilcoxon rank sum test, $n=170$ horizontally and 140 vertically tuned neurons], again similar to findings in the cat (Li et al., 2003). Response amplitudes of orientation-selective neurons (median $\Delta F / F=18.7 \%$ ) did not vary systematically with neurons' preferred orientation.

Stripe-reared mice showed an overrepresentation of the experienced orientation, on average by $64.6 \pm 14.8 \%$, compared with the orthogonal orientation (Fig. 3B-D). This effect was of similar magnitude in the subpopulation of direction-selective neurons and was also reflected in the tuning of the neuropil (data not shown). To quantify the specific effect, we added the increase in the fraction of neurons preferring the experienced orientation to the decrease in preference for the orthogonal orientation (see Materials and Methods). As shown in Figure 3E, the specific effect of stripe rearing was pronounced after stripe rearing with $-45^{\circ}(17.9 \pm 2.9 \%$, $p<0.001), 0^{\circ}(16.7 \pm 4.4 \%, p<0.05)$ and $90^{\circ}(15.7 \pm 4.8 \%, p<0.05)$, but weaker after stripe rearing with $45^{\circ}(9.3 \pm 2.9 \%$, $p<0.05$, all one-sided two-sample $t$ tests, $n=7$ stripe-reared mice each, $n=8$ control mice). We did not observe any significant effect of stripe rearing on the median of response amplitudes and tuning widths (data not shown). Notably, the fraction of nonorientation-selective neurons decreased only after rearing with $45^{\circ}(-9.3 \pm 0.8 \%$ compared with the control group, $p<0.05$, one-sided $t$ test, $n=7$ stripe-reared and 8 control mice).

To test whether experimentally determining orientation preference interferes with the bias induced by stripe rearing (Frenkel et al., 2006; Li et al., 2008), we imaged one test optical section at the beginning and again at the end of most experiments. The average duration of an imaging experiment was $2 \mathrm{~h}$, during which the anesthetized mouse was almost continuously exposed to standing and moving gratings of all orientations. Of a total of 741 neurons detected at the beginning of 25 imaging experiments, we were able to re-identify $78.4 \%$ at the end. For the population of neurons significantly tuned at the start and the end of an experiment we found no net changes in preferred orientation $\left(+0.0 \pm 0.9^{\circ}\right.$, n.s., one-sample $t$ test) for both control and stripe-reared animals. Thus, orientation preference is not altered by measuring it.
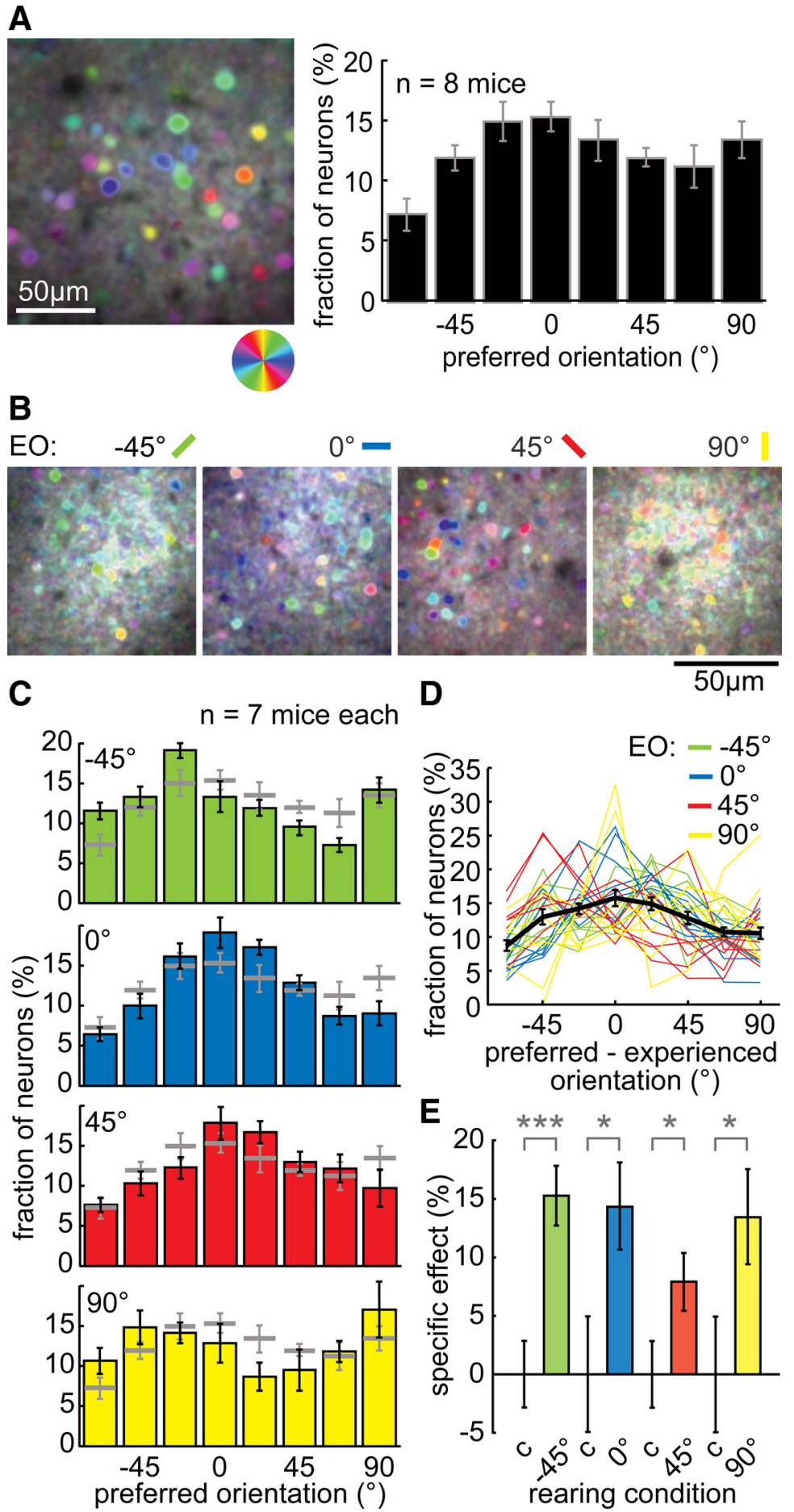

Figure 3. Orientation preference in the primary visual cortex of control and stripe-reared mice. $A$, Orientation preference in control mice raised under standard housing conditions; left: pixel-based orientation map with coding for preferred orientation (hue), response amplitude (lightness) and tuning width (saturation); right: distribution of preferred orientations (mean \pm SEM; $n=8) ;(\boldsymbol{B})$-(E): Orientation preference in stripe-reared mice; (B) Pixel-based orientation maps after stripe rearing with cylinder lenses of $167 \mathrm{dpt}$ for 3 weeks, experienced orientations (E0) are indicated by values and colored bars above maps; (C) Histograms of preferred orientations (mean $\pm \mathrm{SEM} ; n=7$ for each rearing condition), experienced orientations are specified above the graphs, light gray data points: distribution in control mice; (D) Distribution of preferred orientations normalized to the experienced orientation (E0, as indicated by the color code in the legend, set to $0^{\circ}$ ) for 28 stripe-reared mice, black: average distribution (mean $\pm \mathrm{SEM}$ ); $(\boldsymbol{E})$ Average specific effect calculated as the sum of the increase in the fraction of neurons preferring the experienced orientation and the decrease in the fraction of neurons preferring the orthogonal orientation (see Methods) of all four rearing conditions (mean $\pm S E M, n=7$ each) compared with four respective data subsets derived from control (c) mice $(n=8)$. 

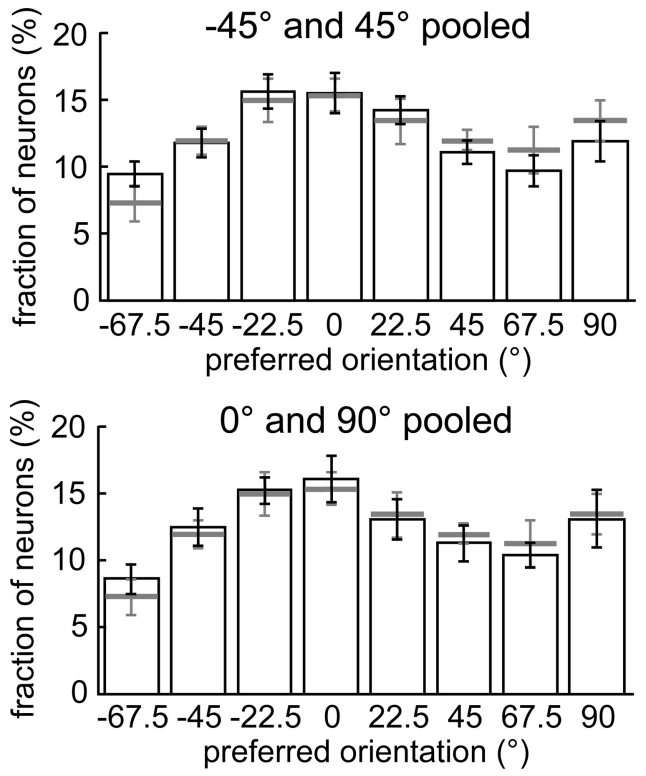

Figure 4. Shifts in distribution of preferred orientations after stripe rearing are complementary. Pooled distribution of preferred orientations of mice reared with orthogonal orientations; top: $-45^{\circ}$ and $45^{\circ}$; bottom: $0^{\circ}$ and $90^{\circ}$; light gray data points: distribution in control mice.

\section{Shifts in orientation preference of orthogonal rearing conditions are complementary}

We next analyzed whether the functional changes induced by different experienced orientations relate to each other in a systematic fashion. Specifically, we asked whether two experienced orientations which are perpendicular would cause complementary modifications of the distribution of preferred orientation or rather lead to common changes. To this end, we calculated the average distribution of preferred orientations of all four stripe rearing conditions. The resulting distribution was very similar to that of the control group (data not shown). The same was true when averaging the distributions of preferred orientations of both orthogonal stripe rearing conditions separately (Fig. 4). This suggests that the shift in the distribution caused by one experienced orientation is complementary to the one that occurs in mice exposed to the orthogonal experienced orientation. The average distributions (Fig. 4) also show that the horizontal bias is preserved in stripe-reared mice, which explains the asymmetric shift of the distributions of preferred orientations; they are biased toward the horizontal orientation as is best visible in mice with oblique experienced orientations (Fig. 3C).

\section{Fraction of responsive neurons is slightly decreased after stripe rearing}

Our results demonstrate a clear effect of stripe rearing in layer $2 / 3$ of mouse visual cortex. A long-standing question has been whether the observed overrepresentation of the experienced orientation can be explained by permissive changes, i.e., changes in responsiveness (rather than orientation preference) of certain neuronal populations (Stryker and Sherk, 1975; Sengpiel et al., 1999). To address this question, we compared the fraction of responsive neurons in control and stripe-reared mice. As the goggle frames occlude part of the visual field, we first tested whether wearing goggles with planar lenses $(0 \mathrm{dpt})$ would result in any kind of visual deprivation effect. Mice with such goggles did not display any difference in the fraction of responsive neurons ( $70 \pm 3 \%, n=7$ mice) compared with control mice $(69 \pm 6 \%, n=$ 8 mice, Fig. 5). We noted that the distribution of preferred

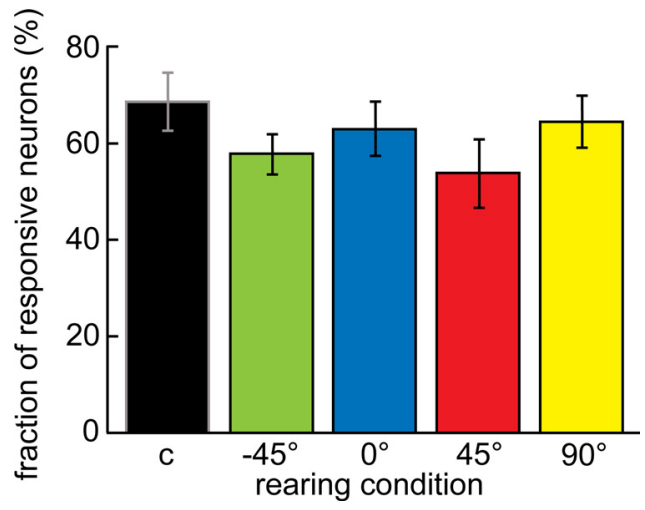

Figure 5. The effect of stripe rearing on the fraction of responsive neurons. Fraction of responsive neurons in control (c) mice and after stripe rearing with different orientation.

A

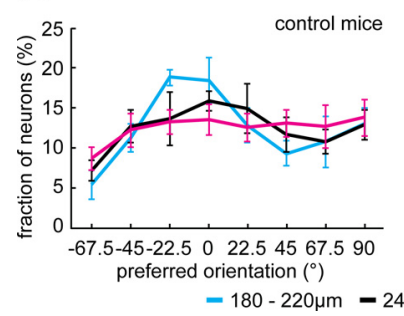

Figure 6. Horizontal bias and permissive effect depend on depth in layer $2 / 3 . A$, Distribution of preferred orientations with increasing depth in layer $2 / 3$ (mean \pm SEM; $n=8$ ), cortical depth is coded in color as indicated at the bottom of the figure; $(\boldsymbol{B})$ Fraction of responsive neurons with increasing depth in layer $2 / 3$.

orientations was slightly flatter than in the control group, but this effect was not significant (two-sample KolmogorovSmirnov test, $p=0.5189$ ).

While there was no difference between control mice and mice wearing goggles with 0 diopter lenses, stripe-reared mice showed a small drop in the overall fraction of responsive neurons $(-11 \pm 4 \%$, $-6 \pm 6 \%,-15 \pm 7 \%$ and $-4 \pm 5 \%$ for rearing with $-45^{\circ}, 0^{\circ}, 45^{\circ}$ and $90^{\circ}$ orientation, respectively, $n=7$ mice each, Fig. 5). This difference was not statistically significant for each individual stripe rearing condition. However, pooling all stripe-reared mice yielded a significant drop in responsiveness $(p=0.0475$, onesided $t$ test, $n=8$ control mice and 28 stripe-reared mice). Overall, the weak tendency toward reduced responsiveness suggests that the stripe rearing procedure may also result in some permissive changes.

\section{Stripe rearing effect varies with depth in layer $2 / 3$ and is} caused by instructive changes in lower layer $2 / 3$

Closer inspection of the distribution of preferred orientations in control mice revealed that the overrepresentation of horizontally tuned neurons (Fig. $3 A$, right) was mainly derived from the upper parts of layer $2 / 3$ and decreased with cortical depth (Fig. $6 \mathrm{~A}$, $180-220 \mu \mathrm{m}$ depth: horizontally tuned: $16.7 \pm 0.9 \%$, vertically tuned: $9.7 \pm 1.1 \%, p<0.0005 ; 240-280 \mu \mathrm{m}$ : horizontal: $14.9 \pm 1.6 \%$, vertical: $10.3 \pm 1.2 \%, p<0.05 ; 300-340 \mu \mathrm{m}$ : horizontal: $13.2 \pm 0.8 \%$, vertical: $11.7 \pm 1.3 \%$, n.s., $t$ test, $n=8$ control mice each). Together with previously reported differences in the degree and developmental time course of plasticity between cortical layers (Trachtenberg et al., 2000; Crozier et al., 2007; Liu et al., 2008), this prompted us to test whether the stripe rearing effect also changed with depth in cortical layer $2 / 3$. We 
found this to be the case: the magnitude of the shift increased with cortical depth. In the uppermost tier $(180-220 \mu \mathrm{m}$ below the cortical surface) the specific effect was small $(8.6 \pm 6.1 \%$, n.s., $t$ test). It increased at the next depth level $(240-280 \mu \mathrm{m}$; $15.5 \pm 2.6 \%, p<0.001, t$ test $)$, and was largest among the bottom cells in layer $2 / 3(300-340 \mu \mathrm{m} ; 18.2 \pm 3.1 \%, p<0.00005, t$ test, $n=28$ stripe-reared and 8 control mice in each case).

Similarly, the changes in responsiveness following stripe rearing depended on cortical depth (Fig. 6B). The fraction of responsive neurons dropped only in the upper $(-18.5 \pm 6.5 \%, p=0.1605)$ and middle tier $(-18.4 \pm 4.0 \%, p<0.05)$ of layer $2 / 3$, which is compatible with a permissive mechanism. In lower layer $2 / 3$, in contrast, the fraction of responsive neurons did not change $(-0.8 \pm 3.3 \%$, $p=0.8935, t$ test, all relative to the fraction of responsive neurons in control mice, $n=28$ stripe-reared and 8 control mice each). This suggests that the comparatively strong stripe rearing effect we observed among neurons in lower layer $2 / 3$ (Fig. 7) is not caused by permissive changes but can be fully accounted for by an instructive mechanism, i.e., a change in the orientation tuning of individual neurons.

\section{Absolute number of neurons tuned to the experienced orientation increases in lower layer $2 / 3$}

To test the latter conjecture in a more direct fashion we computed the number of neurons tuned to a particular orientation as a fraction of the total number of neurons detected. Thereby, we made use of a technical advantage of two-photon calcium imaging, which, unlike electrophysiological recordings, permits the detection of all neurons within a field of view, including the nonresponsive and the non-orientation-selective ones. While the fractions of neurons preferring the experienced or the orthogonal orientation were both strongly decreased in upper layer $2 / 3$ (180-220 $\mu \mathrm{m}$ : experienced orientation: $-3.0 \pm 1.0 \%$, orthogonal orientation: $-4.6 \pm 1.1 \%$, Fig. $8 A$ ), only the fraction of neurons preferring the orthogonal orientation was decreased in the middle tier of layer $2 / 3(240-280 \mu \mathrm{m}$ : experienced orientation: $-1.1 \pm 1.1 \%$, orthogonal orientation: $-6.7 \pm 0.9 \%$, Fig. $8 B$ ). In lower layer $2 / 3(300-340 \mu \mathrm{m})$, the fraction of neurons preferring the orthogonal orientation did not change much $(-2.7 \pm 1.0 \%$, Fig. $8 C$ ), whereas the fraction of neurons preferring the experienced orientation strongly increased by $7.6 \pm 1.5 \%$ (Fig. $8 C$, $n=28$ stripe-reared and 8 control mice each), corresponding to an increase by $40.2 \pm 7.7 \%$ when normalized to the fraction of neurons preferring the experienced orientation in control mice. The effects in middle layer $2 / 3$ are somewhat intermediate to those in upper and lower layer $2 / 3$, which might be explained by either a specific drop in the fraction of responsive neurons implying permissive changes, or by a combination of an unspecific drop in responsiveness as found in upper layer $2 / 3$ and a specific increase in the fraction of neurons preferring the experienced orientation as observed in lower layer $2 / 3$. Together, this analysis demonstrates that the changes caused by stripe rearing are diverse and depend on a neuron's vertical position in the cortex: In upper layer 2/3, we find small effects of stripe rearing; rather, the fraction of orientation-selective neurons is reduced regardless of orientation preference. In lower layer $2 / 3$, there is a clear stripe rearing effect with changes largely due to an instructive mechanism, which leads to shifts in the tuning curves of a large number of neurons.

\section{Can the drop in responsiveness explain the specific effect?}

Those neurons which loose responsiveness in stripe-reared mice are mainly located in upper and middle layer 2/3 (Fig. 6B). Are
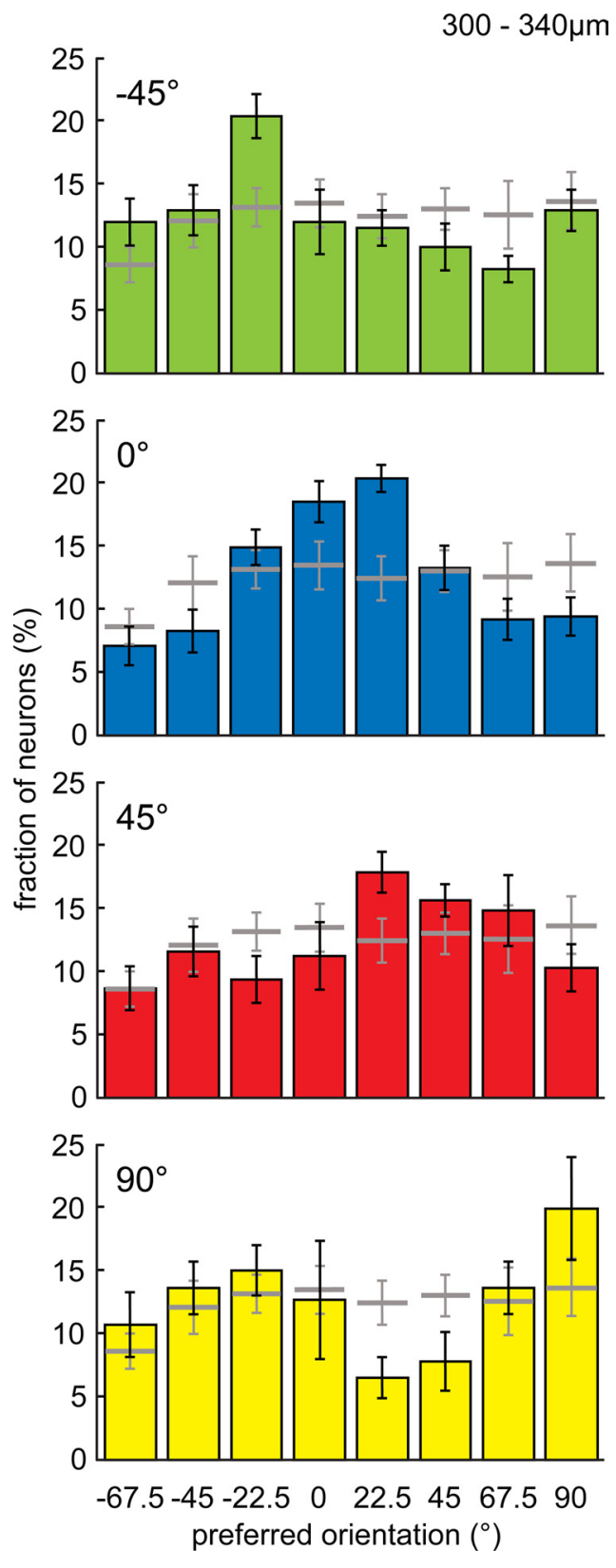

Figure 7. Stripe rearing has a stronger effect on neurons in lower layer $2 / 3$. Distribution of preferred orientations at a depth of 300-340 $\mu \mathrm{m}$ (lower layer 2/3) from stripe-reared mice with experienced orientations of $-45^{\circ}, 0^{\circ}, 45^{\circ}$ and $90^{\circ}$ as indicated; light gray data points: distribution in control mice.

neurons, which prefer the orientation orthogonal to the experienced one, selectively affected by this drop in responsiveness, or does it occur independently of orientation preference? If the first was true, we would expect that stripe-reared mice with a comparatively strong decrease in the fraction of responsive neurons should show an equally stronger overrepresentation of the experienced orientation, quantified as the specific effect. Therefore, we plotted the change in responsiveness as a function of the specific effect for each stripe-reared mouse (Fig. 9). The two parameters were not correlated ( $r=-0.04$, n.s., $n=28$ stripe-reared mice). This was also true when we analyzed data obtained at different cortical depths separately. These results indicate that changes in responsiveness occur, but they are rather unlikely to account for the overrepresentation of the experienced orientation caused by stripe rearing. 
A

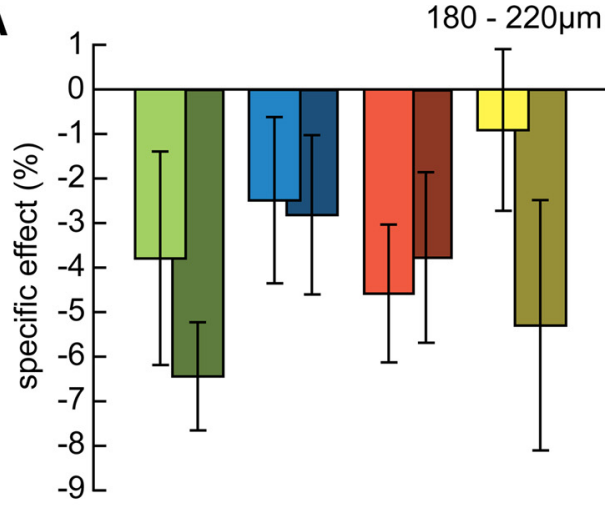

B
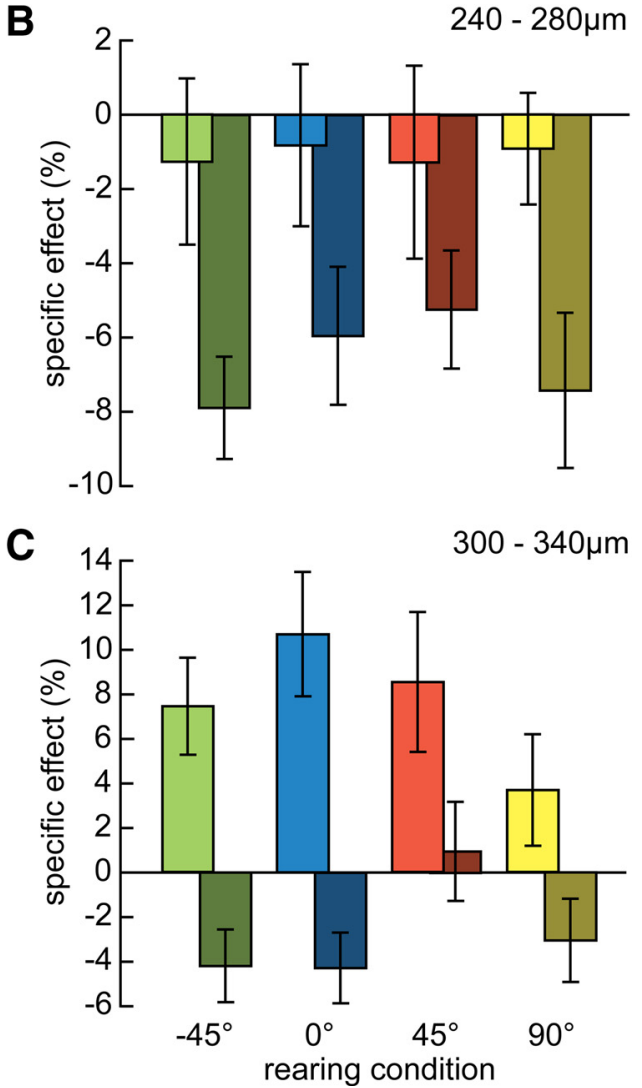

Figure 8. Global specific effect of stripe rearing at different depths of layer 2/3. Global specific effect calculated as percentage of all morphologically detected neurons (see Materials and Methods) derived from the population of orientation-selective neurons normalized to the total number of detected neurons in each animal at (A) $180-220 \mu \mathrm{m},(\boldsymbol{B}) 240-280 \mu \mathrm{m}$ and ( $\boldsymbol{C}$ ) $300-340 \mu \mathrm{m}$; bright bars: experienced orientation, shaded bars: orthogonal orientation.

\section{Stripe rearing effect is stable over a range of temporal and spatial frequencies}

In addition to removing certain contour orientations, the cylinder lenses likely also change the spatial and temporal frequency composition of visual input in stripe-reared mice, which might in turn alter neurons' tuning for these features and render our standard visual stimulus $(0.03 \mathrm{cpd}$ and $1.5 \mathrm{~Hz}$ ) non-optimal. Therefore, we determined orientation preference using a variety of spatial $(0.03,0.04$, 0.06 , and $0.1 \mathrm{cpd})$ and temporal $(1.5,2$ and $3 \mathrm{~Hz})$ frequencies in an additional set of experiments, consisting of four control and eight stripe-reared mice (four with $0^{\circ}$ and four with $-45^{\circ}$ as experienced orientation) at cortical depths of 200 and $300 \mu \mathrm{m}$.

In stripe-reared mice, we reproduced our previous findings: First, the specific effect was large at $300 \mu \mathrm{m}$ and absent at $200 \mu \mathrm{m}$

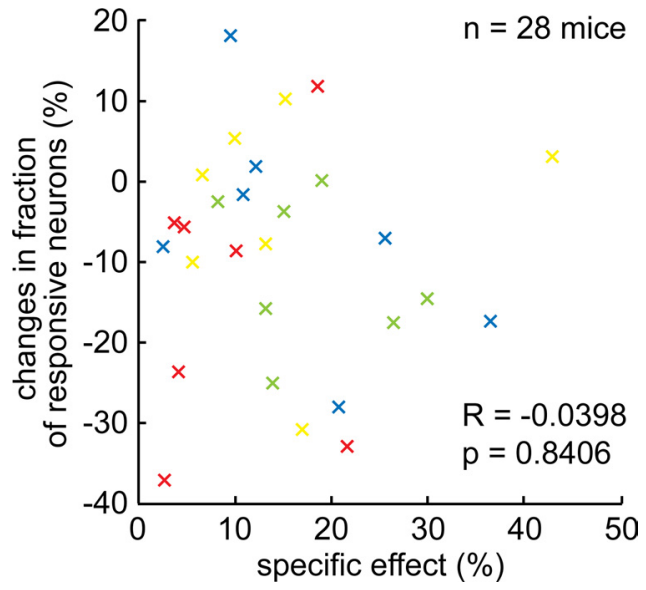

Figure 9. Absence of correlation between permissive and specific stripe rearing effect. Change in the fraction of responsive neurons after stripe rearing as a function of the specific effect for 28 individual mice with experienced orientations as indicated by colors: green $\left(-45^{\circ}\right)$, blue $\left(0^{\circ}\right)$, red $\left(45^{\circ}\right)$ and yellow $\left(90^{\circ}\right)$.
A
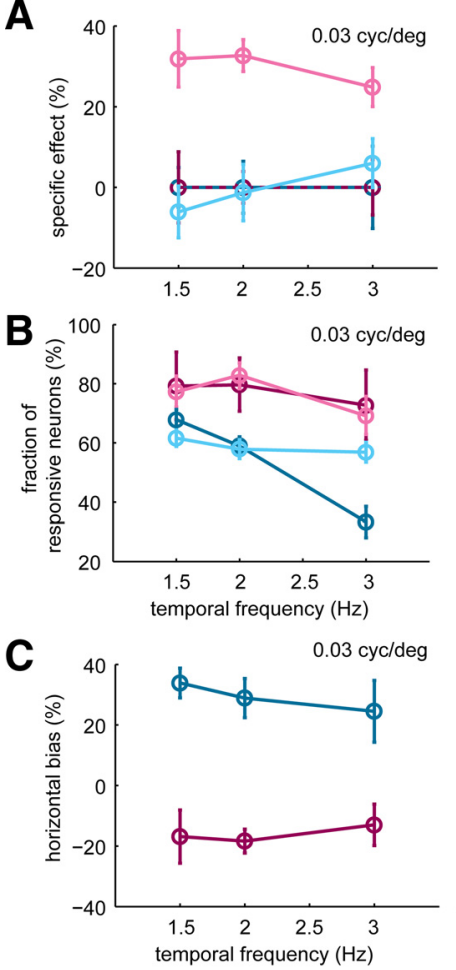

$200 \mu \mathrm{m}$ : - control - stripe reared; $300 \mu \mathrm{m}:$ control - stripe reared
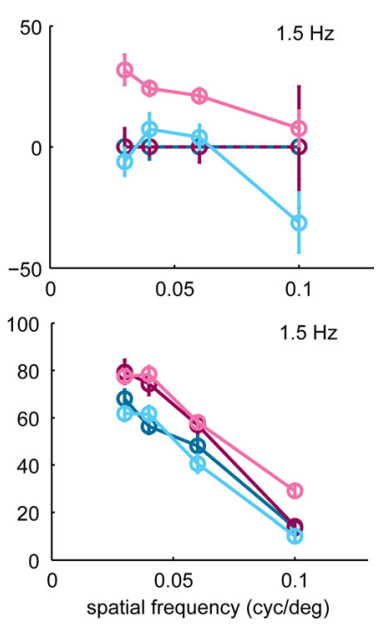

$1.5 \mathrm{~Hz}$

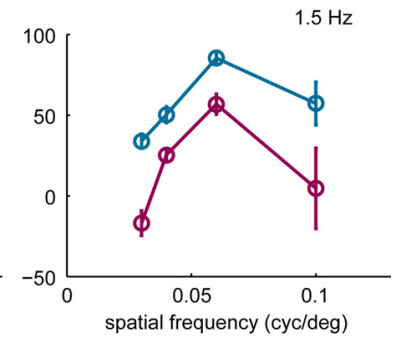

Figure 10. Response properties in stripe-reared and control mice characterized with gratings of different spatial and temporal frequencies. $A, B$, Specific effect $(\boldsymbol{A})$ and fraction of responsive neurons ( $\boldsymbol{B}$ ) at different temporal (left) and spatial (right) frequencies; data were assessed at $200 \mu \mathrm{m}$ (cyan) and $300 \mu \mathrm{m}$ (magenta) in a separate dataset of control (dark) and stripe reared mice (bright). C, Horizontal bias calculated as the difference between the fraction of neurons preferring horizontal and the fraction of neurons preferring vertical orientations (see Materials and Methods) as a function of temporal (left) and spatial frequency (right) at cortical depths of $200 \mu \mathrm{m}$ (dark cyan) and $300 \mu \mathrm{m}$ (dark magenta); data are presented as mean \pm SEM (control mice: $n=4$; mice stripe-reared: $n=8$ in total, 4 each with $0^{\circ}$ and $-45^{\circ}$ experienced orientation, respectively).

cortical depth (Fig. 10A). Second, there was no significant drop in the fraction of responsive neurons, neither at $200 \mu \mathrm{m}$ nor at $300 \mu \mathrm{m}$ depth (Fig. 10B). At the same time, there was also no increase in the fraction of responsive neurons in stripe-reared 
animals at any of the tested stimulus parameters (Fig. 10 B), indicating that the stripe rearing procedure did not shift neuronal response properties toward higher spatial or temporal frequencies on a population level. Importantly, both, specific effect and fraction of responsive neurons were largely independent of temporal and spatial frequency (Fig. 10A,B). An exception is the highest spatial frequency tested $(0.1 \mathrm{cpd})$, where the specific effect appeared to decline, however, only in the rather small fraction of neurons still responsive to this spatial frequency (Fig. $10 \mathrm{~B}$ ). Together, these data support the results from our larger data sample reported above, pointing to an instructive nature of the stripe rearing effect in lower layer $2 / 3$, which does not depend much on the spatial and temporal frequency of the stimulus.

\section{Horizontal bias changes with spatial frequency}

Likewise, we confirmed in control mice that the horizontal bias is larger at $200 \mu \mathrm{m}(33.8 \pm 8.5 \%$ at $0.03 \mathrm{cpd}$ and $1.5 \mathrm{~Hz})$ than at $300 \mu \mathrm{m}(-16.9 \pm 15.2 \%$, both at $0.03 \mathrm{cpd}$ and $1.5 \mathrm{~Hz})$ depth ( $p<0.05$, two-sample $t$ test, $n=4$ mice, Fig. 10C). Changing the temporal frequency did not affect the magnitude of the horizontal bias. However, it strongly increased with increasing the spatial frequency up to $0.06 \mathrm{cpd}$, but decreased again at $0.1 \mathrm{cpd}$ (Fig. 10C). Closer inspection of the changes in response properties of individual neurons depending on the combination of stimulus parameters revealed two components underlying this effect: Partially, the overall increase in horizontal bias was caused by the recruitment of neurons preferring higher spatial frequencies (7.4\% of the neurons responding at $0.04 \mathrm{cpd}$ were newly recruited compared with $0.03 \mathrm{cpd}$ and $14.6 \%$ of the neurons responding at $0.06 \mathrm{cpd}$ were newly recruited compared with $0.04 \mathrm{cpd})$. Most of these were tuned to horizontal orientations $(52.6 \%$ at $0.04 \mathrm{cpd}$ and $78.1 \%$ at $0.06 \mathrm{cpd}$ ). Partially, the effect can be attributed to shifts in preferred orientation when changing the spatial frequency of the stimulus: Of all neurons responsive at $0.04 \mathrm{cpd}$, $65.9 \%$ changed their preferred orientation toward horizontal by on average $19.4 \pm 1.6^{\circ}$ and $26.7 \%$ changed their preferred orientation toward vertical by on average $17.8 \pm 3.0^{\circ}$ compared with $0.03 \mathrm{cpd}$. Likewise, of all neurons responsive at $0.06 \mathrm{cpd}, 71.8 \%$ changed their preferred orientation toward horizontal by on average $17.8 \pm 1.5^{\circ}$ and $13.6 \%$ changed their preferred orientation toward vertical by on average $14.3 \pm 4.0^{\circ}$ compared with $0.04 \mathrm{cpd}$. This seems to be at odds with studies in cat visual cortex, which reported preferred orientation to be independent from stimulus spatial frequency (Webster and De Valois, 1985; Jones et al., 1987). However, large shifts in orientation preference $\left(>10^{\circ}\right)$ occurred in $\sim 70 \%$ of cases in neurons with low response amplitudes $(<11 \%$ $\Delta F / F)$, in which the determination of preferred orientation is more error-prone.

\section{Discussion}

This study for the first time describes how stripe rearing affects the response properties of single neurons in mouse visual cortex. Limiting visual experience to contours of a single orientation for 3 weeks caused a modest overrepresentation of the experienced orientation. Instructive modifications as well as a small drop in responsiveness were observed. The stripe rearing effect had a clear instructive component in lower layer $2 / 3$ as indicated by an increase in the absolute number of neurons tuned to the experienced orientation. Thus, for this subpopulation of neurons in mouse visual cortex, the visual environment acts as an instructive signal, which changes the orientation preference of single neurons in an adaptive fashion.

Almost all stripe rearing experiments to date have been performed in kittens (Blakemore and Cooper, 1970; Stryker et al.,
1978; Sengpiel et al., 1999). The present experiments have been done in mouse primary visual cortex, where neurons also show selective responses to a variety of visual stimulus parameters like visual field position, spatial and temporal frequency, and direction of motion (Dräger, 1975; Niell and Stryker, 2008). Importantly, many neurons are orientation-selective, and their tuning widths are comparable to those of neurons in higher mammals such as cats and ferrets (Niell and Stryker, 2008). While orientation preference in rodents is not organized into a continuous map, but rather forms a "salt-and-pepper" arrangement (Dräger, 1975; Ohki et al., 2005; Sohya et al., 2007), we observed further similarities in response properties of neurons in mouse, ferret (Coppola et al., 1998a), and cat visual cortex (Li et al., 2003), such as the dependence of tuning width on orientation preference, and the presence of a horizontal bias, which was previously noted in the mouse (Dräger, 1975; Frenkel et al., 2006). We in addition observed a change in the distribution of preferred orientations within a few hundred micrometers: In upper layer $2 / 3$ we found a strong overrepresentation of horizontally tuned neurons, while the distribution of preferred orientations was nearly flat in lower layer $2 / 3$ using standard stimulus parameters. This might be an intrinsic property, possibly caused by layer-specific termination of input from specific subsets of retinal ganglion cells relayed via the lateral geniculate nucleus (Kim et al., 2008; Huberman et al., 2009; Kay et al., 2011).

Unlike most previous stripe rearing studies, we did not interfere with the early development of response properties in visual cortex, but rather induced plasticity of orientation preference at a slightly later stage. Therefore, instead of being dark-reared, mice had normal visual experience until postnatal day (p) 25, at which time orientation selectivity is well established, though not completely mature (Wang et al., 2010). On the one hand, this might limit the extent of plasticity (Rauschecker, 1982), on the other hand, it allows dissociating functional impairment by abnormal visual cortex development from plasticity effects (Freeman and Pettigrew, 1973; Frégnac and Imbert, 1978; Gordon and Presson, 1982). Stripe rearing was performed for $\sim 3$ weeks, thus including the peak of the critical period for ocular dominance plasticity around P28, at which time also orientation selectivity has been shown to be highly plastic (Wang et al., 2010).

In our experiments in mouse visual cortex, the experienced orientation was overrepresented after stripe rearing, similar to results previously obtained in kittens (Blakemore and Cooper, 1970; Hirsch and Spinelli, 1970; Blasdel et al., 1977; Stryker et al., 1978; Sengpiel et al., 1999; Tanaka et al., 2007) and rats (O'Hashi et al., 2007). The specific effect, i.e., the sum of the increase in the experienced and the decrease in the orthogonal orientation, was on average $15 \%$. The magnitude of the effect is thus similar to that found in kittens (Sengpiel et al., 1999), showing that mice display a considerable degree of orientation plasticity (Wang et al., 2010), even after previous normal visual experience.

The ability to image all neurons, including the non-responsive ones, allowed us to test two ideas that have been put forward to underlie the stripe rearing effect, instructive and permissive ones. While the permissive hypothesis describes experience-dependent plasticity as a passive process, mainly caused by input loss similar to deprivation, the instructive hypothesis assumes remodeling of circuitry, thereby causing a change in response properties of single neurons. These two mechanisms are not mutually exclusive, but could occur in combination (Rauschecker and Singer, 1981). The importance of permissive changes for the stripe rearing effect has been under debate for decades (Stryker et al., 1978; Rauschecker and Singer, 1981; Sengpiel et al., 1999), but its exact 
contribution has been hard to quantify, due to the lack of adequate sampling methods. With two-photon microscopy we were able to quantify, in an unbiased fashion, the fraction of responsive neurons.

Stripe rearing did cause a slight drop in responsiveness, but the magnitude of this effect was unevenly distributed across the depth of layer $2 / 3$. While a loss of $\sim 10 \%$ in responsiveness occurred in upper and middle layer $2 / 3$, it was absent in lower layer $2 / 3$. It has been shown previously that orientation preference plasticity as well as other types of plasticity in the visual cortex vary in their time course and extent between layers (Singer et al., 1981; Tieman and Hirsch, 1982; Trachtenberg et al., 2000; De Paola et al., 2006; Crozier et al., 2007; Liu et al., 2008) or even sublayers (Holtmaat et al., 2006). Still, this variability within one layer is remarkable. Previous results indicated that the effect of stripe rearing depends on neuronal subtype and is especially weak in neurons with small receptive fields (Hirsch et al., 1983). However, what functionally distinguishes neurons located in upper or lower layer $2 / 3$ in mouse visual cortex remains to be determined.

The weak decrease in responsiveness upon stripe rearing, in the order of $10 \%$ on average, would in principle be compatible with a permissive mechanism. However, in upper layer $2 / 3$, changes in both responsiveness and selectivity affected neurons regardless of their preferred orientation, largely independent of the experienced orientation. Thus, while stripe rearing reduces overall cortical responsiveness to some degree, its contribution to the shift in orientation preference seems rather small. This is further strengthened by the observation that there is no correlation between change in responsiveness and stripe rearing effect.

In contrast to permissive mechanisms, instructive changes were critical for the stripe rearing effect, at least in the cortical layers we observed. This was most obvious in lower layer $2 / 3$, where permissive effects were absent and the total number of neurons preferring the experienced orientation increased strongly. Thus, the stripe rearing effect in this population of neurons is entirely explained by an instructive mechanism. In other populations of neurons this effect was less pronounced. Using temporally precise pairing between an oriented visual stimulus and electrical stimulation in the visual cortex (Schuett et al., 2001), it was shown that neurons can in principle undergo instructive changes of preferred orientation when activated appropriately. Under more physiological conditions, presentation of moving gratings immediately after eye opening (Li et al., 2008), repeated presentation of a single orientation to awake mice (Frenkel et al., 2006) and learning an orientation discrimination task (Schoups et al., 2001) instructively changed neuronal response properties in primary visual cortex. Proving that neurons change their preferred orientation during stripe rearing, our data demonstrate that instructive mechanisms also contribute to experience-dependent orientation plasticity.

In summary, visual experience is capable of modifying orientation selectivity in an instructive manner. A priori both, instructive and permissive changes could have caused the well known effects of stripe rearing. We now demonstrate that instructive changes play the main role in layer $2 / 3$, obviously not precluding that this could be different in deeper layers that we have not imaged. Still, for the upper layers, this indicates that neurons not driven by stimuli present in the sensory environment are not simply silenced; rather, their response properties actively adapt to the specific input-in view of optimally using resources and optimizing performance certainly an advantageous strategy. This assumption is underlined by the following three arguments: First, the response properties of neurons are fine-tuned during devel- opment (Chapman and Bonhoeffer, 1998; Coppola et al., 1998a; Müller et al., 2000), until their distribution matches natural scene statistics with its bias of cardinal (Coppola et al., 1998b), and in particular horizontal orientations (Betsch et al., 2004). Second, an increased number of neurons preferring a certain orientation seems to facilitate more precise discrimination in the overrepresented orientation range (Hirsch, 1972; Wark and Peck, 1982). This relationship becomes most obvious in the so-called "oblique effect," which terms the better performance at cardinal compared with oblique orientations in a number of measurements, such as spatial acuity, contrast sensitivity, or orientation discrimination. Li et al. (2003) described the overrepresentation of cardinal orientations in cat visual cortex as its neural basis. Finally, an optimal representation of orientations in the brain should help facilitating the rapid detection of relevant at the expense of less important features. Such functional specialization can also be observed in humans, for example as facilitated reading of italic letters (Whitaker and McGraw, 2000). All these phenomena point to an instructive, experience-dependent process, continuously shaping the sensory brain paths with the benefit of improved perception that allows for a proper reaction to challenges in an altering environment.

\section{References}

Antonini A, Fagiolini M, Stryker MP (1999) Anatomical correlates of functional plasticity in mouse visual cortex. J Neurosci 19:4388-4406.

Betsch BY, Einhäuser W, Körding KP, König P (2004) The world from a cat's perspective-statistics of natural videos. Biol Cybern 90:41-50.

Blakemore C, Cooper GF (1970) Development of brain depends on visual environment. Nature 228:477-478.

Blakemore C, Van Sluyters RC (1975) Innate and environmental factors in development of kittens visual-cortex. J Physiol-London 248:663-716.

Blasdel GG, Mitchell DE, Muir DW, Pettigrew JD (1977) Physiological and behavioral study in cats of effect of early visual experience with contours of a single orientation. J Physiol 265:615-636.

Chapman B, Bonhoeffer T (1998) Overrepresentation of horizontal and vertical orientation preferences in developing ferret area 17. Proc Natl Acad Sci U S A 95:2609-2614.

Coppola DM, White LE, Fitzpatrick D, Purves D (1998a) Unequal representation of cardinal and oblique contours in ferret visual cortex. Proc Natl Acad Sci U S A 95:2621-2623.

Coppola DM, Purves HR, McCoy AN, Purves D (1998b) The distribution of oriented contours in the real word. Proc Natl Acad Sci USA 95:4002-4006.

Crair MC, Gillespie DC, Stryker MP (1998) The role of visual experience in the development of columns in cat visual cortex. Science 279:566-570.

Crozier RA, Wang Y, Liu CH, Bear MF (2007) Deprivation-induced synaptic depression by distinct mechanisms in different layers of mouse visual cortex. Proc Natl Acad Sci U S A 104:1383-1388.

De Paola V, Holtmaat A, Knott G, Song S, Wilbrecht L, Caroni P, Svoboda K (2006) Cell type-specific structural plasticity of axonal branches and boutons in the adult neocortex. Neuron 49:861-875.

Dräger UC (1975) Receptive fields of single cells and topography in mouse visual cortex. J Comp Neurol 160:269-290.

Freeman RD, Pettigrew JD (1973) Alteration of visual cortex from environmental asymmetries. Nature 246:359-360.

Frégnac Y, Imbert M (1978) Early development of visual cortical cells in normal and dark-reared kittens: relationship between orientation selectivity and ocular dominance. J Physiol 278:27-44.

Frenkel MY, Sawtell NB, Diogo AC, Yoon B, Neve RL, Bear MF (2006) Instructive effect of visual experience in mouse visual cortex. Neuron 51:339-349.

Gordon B, Presson J (1982) Orientation deprivation in cat: what produces the abnormal cells. Exp Brain Res 46:144-146.

Gordon JA, Stryker MP (1996) Experience-dependent plasticity of binocular responses in the primary visual cortex of the mouse. J Neurosci 16:3274-3286.

Hirsch HV (1972) Visual perception in cats after environmental surgery. Exp Brain Res 15:405-423. 
Hirsch HV, Spinelli DN (1970) Visual experience modifies distribution of horizontally and vertically oriented receptive fields in cats. Science 168:869-871.

Hirsch HV, Leventhal AG, McCall MA, Tieman DG (1983) Effects of exposure to lines of one or two orientations on different cell-types in striate cortex of cat. J Physiol 337:241-255.

Hofer SB, Mrsic-Flogel TD, Bonhoeffer T, Hübener M (2006) Prior experience enhances plasticity in adult visual cortex. Nat Neurosci 9:127-132.

Holtmaat A, Wilbrecht L, Knott GW, Welker E, Svoboda K (2006) Experience-dependent and cell-type-specific spine growth in the neocortex. Nature 441:979-983.

Hubel DH, Wiesel TN (1970) The period of susceptibility to the physiological effects of unilateral eye closure in kittens. J Physiol 206:419-436.

Huberman AD, Wei W, Elstrott J, Stafford BK, Feller MB, Barres BA (2009) Genetic identification of an On-Off direction-selective retinal ganglion cell subtype reveals a layer-specific subcortical map of posterior motion. Neuron 62:327-334.

Jones JP, Stepnoski A, Palmer LA (1987) The two-dimensional spectral structure of simple receptive fields in cat striate cortex. J Neurophysiol 58:1212-1232.

Kay JN, De la Huerta I, Kim IJ, Zhang Y, Yamagata M, Chu MW, Meister M, Sanes JR (2011) Retinal ganglion cells with distinct directional preferences differ in molecular identity, structure, and central projections. J Neurosci 31:7753-7762.

Kim IJ, Zhang Y, Yamagata M, Meister M, Sanes JR (2008) Molecular identification of a retinal cell type that responds to upward motion. Nature 452:478-482.

Li B, Peterson MR, Freeman RD (2003) Oblique effect: a neural basis in the visual cortex. J Neurophysiol 90:204-217.

Li Y, Van Hooser SD, Mazurek M, White LE, Fitzpatrick D (2008) Experience with moving visual stimuli drives the early development of cortical direction selectivity. Nature 456:952-956.

Liu CH, Heynen AJ, Shuler MG, Bear MF (2008) Cannabinoid receptor blockade reveals parallel plasticity mechanisms in different layers of mouse visual cortex. Neuron 58:340-345.

Müller T, Stetter M, Hübener M, Sengpiel F, Bonhoeffer T, Gödecke I, Chapman B, Löwel S, Obermayer K (2000) An analysis of orientation and ocular dominance patterns in the visual cortex of cats and ferrets. Neural Comput 12:2573-2595.

Niell CM, Stryker MP (2008) Highly selective receptive fields in mouse visual cortex. J Neurosci 28:7520-7536.

Nimmerjahn A, Kirchhoff F, Kerr JN, Helmchen F (2004) Sulforhodamine 101 as a specific marker of astroglia in the neocortex in vivo. Nat Methods $1: 31-37$.

O’Hashi K, Miyashita M, Tanaka S (2007) Experience-dependent orientation plasticity in the visual cortex of rats chronically exposed to a single orientation. Neurosci Res 58:86-90.

Ohki K, Chung S, Ch'ng YH, Kara P, Reid RC (2005) Functional imaging with cellular resolution reveals precise micro-architecture in visual cortex. Nature 433:597-603.

Rauschecker JP (1982) Instructive changes in the kittens visual cortex and their limitation. Exp Brain Res 48:301-305.
Rauschecker JP, Singer W (1981) The effects of early visual experience on the cats visual cortex and their possible explanation by Hebb synapses. J Physiol 310:215-239.

Sawtell NB, Frenkel MY, Philpot BD, Nakazawa K, Tonegawa S, Bear MF (2003) NMDA receptor-dependent ocular dominance plasticity in adult visual cortex. Neuron 38:977-985.

Schoups A, Vogels R, Qian N, Orban G (2001) Practising orientation identification improves orientation coding in V1 neurons. Nature 412:549-553.

Schuett S, Bonhoeffer T, Hübener M (2001) Pairing-induced changes of orientation maps in cat visual cortex. Neuron 32:325-337.

Sengpiel F, Stawinski P, Bonhoeffer T (1999) Influence of experience on orientation maps in cat visual cortex. Nat Neurosci 2:727-732.

Singer W, Freeman B, Rauschecker J (1981) Restriction of visual experience to a single orientation affects the organization of orientation columns in cat visual-cortex. A study with deoxyglucose. Exp Brain Res 41:199-215.

Sohya K, Kameyama K, Yanagawa Y, Obata K, Tsumoto T (2007) GABAergic neurons are less selective to stimulus orientation than excitatory neurons in layer II/III of visual cortex, as revealed by in vivo functional $\mathrm{Ca}^{2+}$ imaging in transgenic mice. J Neurosci 27:2145-2149.

Stosiek C, Garaschuk O, Holthoff K, Konnerth A (2003) In vivo two-photon calcium imaging of neuronal networks. Proc Natl Acad Sci U S A 100: 7319-7324.

Stryker MP, Sherk H (1975) Modification of cortical orientation selectivity in the cat by restricted visual experience: a reexamination. Science 190:904-906.

Stryker MP, Sherk H, Leventhal AG, Hirsch HV (1978) Physiological consequences for cats visual cortex of effectively restricting early visual experience with oriented contours. J Neurophysiol 41:896-909.

Tagawa Y, Kanold PO, Majdan M, Shatz CJ (2005) Multiple periods of functional ocular dominance plasticity in mouse visual cortex. Nat Neurosci 8:380-388.

Tanaka S, Tani T, Ribot J, Yamazaki T (2007) Chronically mountable goggles for persistent exposure to single orientation. J Neurosci Methods 160:206-214.

Tieman SB, Hirsch HV (1982) Exposure to lines of only one orientation modifies dendritic morphology of cells in the visual cortex of the cat. J Comp Neurol 211:353-362.

Trachtenberg JT, Trepel C, Stryker MP (2000) Rapid extragranular plasticity in the absence of thalamocortical plasticity in the developing primary visual cortex. Science 287:2029-2032.

Wang BS, Sarnaik R, Cang J (2010) Critical period plasticity matches binocular orientation preference in the visual cortex. Neuron 65:246-256.

Wark RC, Peck CK (1982) Behavioral consequences of early visual exposure to contours of a single orientation. Dev Brain Res 281:218-221.

Webster MA, De Valois RL (1985) Relationship between spatial-frequency and orientation tuning of striate-cortex cells. J Opt Soc Am A 2:1124-1132.

Whitaker D, McGraw PV (2000) Long-term visual experience recalibrates human orientation perception. Nat Neurosci 3:13.

White LE, Coppola DM, Fitzpatrick D (2001) The contribution of sensory experience to the maturation of orientation selectivity in ferret visual cortex. Nature 411:1049-1052. 